\title{
Microencapsulação de probióticos pelo método de extrusão associado a interações eletrostáticas
}

\author{
Microencapsulation of probiotics by extrusion method associated with electrostatic \\ interactions
}

\author{
Mariana de Araújo Etchepare ${ }^{1^{*}}$, Maria Fernanda da Silveira Cáceres de Menezes ${ }^{1}$, Andressa \\ Ribas Barreto ${ }^{2}$, Carlos Pasqualin Cavalheiro ${ }^{1}$, Cristiano Ragagnin de Menezes ${ }^{3}$ \\ ${ }^{1}$ Doutorandos em Ciência e Tecnologia de Alimentos, Universidade Federal de Santa Maria, RS, Brasil \\ ${ }^{2}$ Graduanda em Tecnologia de Alimentos, Universidade Federal de Santa Maria, RS, Brasil \\ ${ }^{3}$ Professor Doutor, Universidade Federal de Santa Maria, RS, Brasil
}

\begin{abstract}
Resumo
Probióticos são microrganismos vivos que quando consumidos em quantidades adeequadas conferem uma série de benefícios para a saúde do hospedeiro devido à melhora da microflora intestinal. Bactérias do gênero Lactobacillus, Bifidobacterium, e Bacillus têm sido amplamente pesquisadas e são adicionadas em diversos tipos de alimentos. No entanto, a estabilidade físico-química e biodisponibilidade destas bactérias têm representado um grande desafio para a indústria de alimentos, principalmente em produtos alimentares não refrigerados. A microencapsulação ajuda a melhorar a sobrevivência destas bactérias, pois protege os microrganismos de condições adversas, tais como altas e baixas temperaturas, $\mathrm{pH}$, aditivos, ou, durante o processamento e sua passagem pelo trato gastrointestinal. A maioria dos materiais de revestimento usados na microncapsulação de probióticos para veicular alimentos são polissacarídeos microbianos iónicos, exopolissacarídeos, e proteinas do leite, os quais exibem diferentes características físico-químicas. A preparação das microcápsulas resulta de inúmeras técnicas disponíveis, cada uma com sua particularidade. Dentre as técnicas de microencapsulação de probióticos, a extrusão é a mais popularmente empregada para a obtenção de micropartículas. Nesta revisão, serão abordados tópicos a respeito da microencapsulação de probióticos pelo método de extrusão associado a interações eletrostáticas para o desenvolvimento de microcápsulas com potencial aplicação em alimentos.
\end{abstract}

Palavras-chave: Probióticos. Microencapsulação. Extrusão. Interações eletrostáticas. Alimentos

\begin{abstract}
Probiotics are live microorganisms that when consumed in adeequadas amounts confer a number of benefits for the health of the host due to improved intestinal microflora. Bacteria of the genus Lactobacillus, Bifidobacterium, and Bacillus have been widely studied and are added to various food types. However, the physicochemical stability and bioavailability of these bacteria have represented a major challenge for the food industry, especially in non-refrigerated food products. The microencapsulation helps to improve the survival of these bacteria, it protects the microorganisms from adverse conditions such as high and low temperatures, $p H$, additives, or during processing and its passage through the gastrointestinal tract. Most flooring materials used in the carrier to microncapsulação probiotic foods are ionic microbial polysaccharides, exopolysaccharides, and milk proteins, which exhibit different physicochemical characteristics. The preparation of microcapsules results from numerous techniques available, each with its particularity. Among the microencapsulation techniques probiotics, extrusion is most popularly employed for obtaining microparticles. In this review, will be addressed topics regarding microencapsulation of probiotics by extrusion method associated with electrostatic interactions to develop microcapsules with potential application in foods.
\end{abstract}

Keywords: Probiotics. Microencapsulation. Extrusion. Electrostatic interactions. Food 


\section{Introdução}

No início do século 20, o cientista russo Élie Metchnikoff propôs que a saúde, o bemestar e a longevidade das populações balcânicas eram atribuíveis ao consumo de grandes quantidades de leite fermentado, contendo microrganismos benéficos denominados de probióticos. Após diversas definições propostas, a definição mais atual refere-se ao conceito de que probióticos são organismos vivos que, quando administrados em quantidades adequadas conferem uma série de benefícios à saúde do hospedeiro, cuja ingestão leva a importantes mudanças fisiológicas ao organismo, através do equilíbrio da microbiota intestinal (Corona-Hernandez et al, 2013; FAO, 2001).

Inúmeros benefícios à saúde têm sido atribuídos à ingestão de probióticos, porém, é importante ressaltar que tais benefícios somente ocorrem se os mesmos estiverem totalmente viáveis nos produtos onde serão incluídos, ou seja, que sobrevivam durante seu processamento e condições de armazenamento, sendo assim ingeridos em quantidades adequadas, alcançando um número viável de microrganismos (10 $\mathrm{UFC}^{-1}$ ) (Mortazavian et al. 2007). No Brasil, a legislação recomenda $10^{8}-10^{9}$ UFC/100g na recomendação diária do produto pronto para consumo (Brasil, 2012a).

As principais espécies probióticas pertencem aos gêneros Lactobacillus e Bifidobacterium que fazem parte da microbiota do intestino humano, pois exercem efeitos benéficos para a saúde humana e melhoram as propriedades da microbiota nativa (Shah, 2007; Menezes; Durrant, 2008; Cook et al., 2012).

Nos países desenvolvidos é crescente a popularidade dos alimentos funcionais contendo probióticos e isto se deve aos avanços nas pesquisas em desenvolvimento de novos produtos, que resultaram na incorporação de probióticos não só em produtos lácteos, mas também em bebidas, produtos de panificação, maionese, sopas de legumes, cereais, pastas de azeitona e até mesmo em produtos cárneos (Altamirano-Fortoul et al., 2012; Trujillo-de
Santiago et al., 2012; Zanjani et al., 2012; Gawkowski; Chikindas 2013; Mattila-Sandholm, 2002; Fahimdanesh et al., 2012; Barbosa et al., 2012, Alves et al. 2015).

No entanto, à diversidade de características intrínsecas e extrínsecas dos alimentos, aliadas às várias alterações físicoquímicas que ocorrem durante o processamento e armazenamento de alimentos probióticos, podem ser um fator negativo para manter a viabilidade dos microrganismos nestes produtos. Devido a isso, várias tecnologias estão sendo empregadas para garantir a sobrevivência dos probióticos dentro de laticínios e de novas matrizes alimentares (Gomes et al., 2011; Ibarra et al., 2012), como por exemplo, a microencapsulação.

A microencapsulação de probióticos tem como objetivo, proteger os microrganismos frente a meio ácidos, sais biliares, oxigênio, altas temperaturas, e no tempo de armazenamento (Oliveira et al., 2007a; Souza et al; 2015; Fareez et al. 2015).

Para fornecer a proteção e a sobrevivência das bactérias probióticas durante o armazenamento e digestão, diferentes métodos de microencapsulação (extrusão, emulsão, secagem por pulverização, entre outros) têm sido desenvolvido, para que os probióticos sejam retidos numa matriz reconhecida como segura (GRAS) (geralmente alginato, carragena, gomas, pectina, quitosana, inulina, proteínas do soro do leite) e insolúvel para garantir a integridade do microrganismo, tanto no alimento como na parte superior do trato gastrointestinal (Krasaekoopt, Bhandari, \& Deeth, 2003; Nazzaro, Orlando, Fratianni, \& Coppola, 2012; Krasaekoopt e Watcharapoka, 2014).

Um dos métodos de microencapsulação que vem sendo bastante utilizado é o de extrusão. Este método apresenta condições simples e acessíveis para a produção das micropartículas, não utiliza temperatura e solvente, tornando-o promissor e muito utilizado (De Prisco et al 2015; Ribeiro et al, 2014; Krasaekoopt et al., 2003).

Este artigo de revisão irá abordar tópicos de microencapsulação de probióticos com ênfase 
no método de extrusão combinado com interações eletrostáticas.

\section{Microencapsulação de probióticos}

A microencapsulação tem sido utilizada com a finalidade de proteger as bactérias probióticas das condições adversas do trato digestivo e também no alimento durante sua vida útil. (Champagne; Fustier, 2007; Argin et al. 2014). Estudos específicos para verificar a proteção das bactérias microencapsuladas em relação a bactérias livres contra a exposição a oxigênio, baixas temperaturas de congelamento, suco gástrico, meios ácidos, estocagem e tratamento térmico já trazem resultados promissores (Kim \& Olsom, 1989; Sheu \& Marshal, 1993; Albertini et al. 2010; Etchepare et al. 2016; Kim et al. 2008)

$\mathrm{Na}$ indústria de alimentos diversos polissacarídeos, proteínas e lipídios são utilizados no desenvolvimento de microcápsulas. A utilização de proteínas e/ou polissacarídeos depende de alguns fatores, como a capacidade dos biopolímeros em formar partículas, serem atóxicos e moduláveis a ponto de se produzir partículas com as características desejadas, com tamanho, estrutura, carga, permeabilidade e estabilidade desejados (Souza et al. 2015).

O uso de hidrocolóides para formação de partículas de géis tem apresentado crescente interesse por diversas áreas de aplicação, tais como alimentícia, farmacêutica e cosmética (Burey et al., 2008). Estes hidrocolóides são particularmente atraentes por suas propriedades de formar géis e alterar a viscosidade quando dispersos em meio aquoso como o alginato, por exemplo, que tem sido empregado como material encapsulante devido a sua capacidade para absorver água, ser de fácil manipulação e inocuidade, possuindo ainda outras características como propriedades gelificantes, estabilizantes e espessantes, razões pelas quais tem sido de grande interesse para a indústria de alimentos (Pasin, Azón \& Garriga, 2012).

Entre os sais de alginato mais empregados, se encontra o alginato de sódio, devido a sua alta solubilidade em água fria e características de transição de solução para gel de forma instantânea quando em contato com íons cálcio, sendo o material encapsulate mais utilizado para o método de extrusão (Funami et al., 2009; Etchepare et al., 2016).

O alginato de sódio é o polissacarídio mais utilizado como material encapsulante de bactérias ácido lácticas, devido a facilidade de manejo, natureza não tóxica e baixo custo (Sultana et al., 2003; Etchepare et al. 2016), aumentando a viabilidade dessas bactérias quando submetidas a diferentes condições ao serem comparadas com bactérias não encapsuladas (De Prisco et al. 2015; Alves et al., 2015; Albadran et al. 2015).

Diversos métodos podem ser utilizados para a microencapsulação de probióticos e a escolha deste, depende da aplicação que será dada à microcápsula, o tamanho que se deseja alcançar, o mecanismo de liberação e propriedades físico-químicas, tanto do material ativo, quanto do agente encapsulante (Jackson; Lee, 1991; Souza et al. 2015).

Lambert et al., (2008) afirmam que algumas técnicas disponíveis, tais como secagem por atomização ou envolvimento lipossômico, podem não ser adequadas para a microencapsulação de bactérias probióticas, pois requerem o emprego de altas temperaturas ou solventes orgânicos, o que pode ser prejudicial aos microrganismos. No entanto, a utilização de outras técnicas de encapsulação como, por exemplo, a extrusão pode conferir maior proteção aos microrganismos encapsulados em função das condições utilizadas para a produção das micropartículas (Krasaekoopt et al., 2003; Etchepare et al. 2016).

\section{Extrusão / Gelificação Iônica}

Dentre as técnicas de microencapsulação de probióticos, a extrusão é a mais popularmente empregada para a obtenção de micropartículas de alginato de cálcio (Pedroso, 2011; De Prisco et al., 2015; Ribeiro et al. 2014).

O método físico baseia-se na gelificação externa do alginato e consiste em incorporar o material a encapsular numa solução de alginato de sódio, para depois a mistura sofrer extrusão gota a gota, através de uma pipeta de calibre reduzido ou de uma seringa, para uma solução de cloreto de cálcio $\left(\mathrm{CaCl}_{2}\right)$ (Smrdel et al., 2008). As partículas devem permanecer em torno de 20 minutos em contato com a solução iônica para atingir estabilidade e resistência mecânica, alem do mais, o tempo que estas partículas 
permanecem nesta solução está diretamente relacionado com a espessura da parede da cápsula e conseqüentemente com a sua resistência a ruptura mecânica (Racoviță et al., 2009; Pasqualim et al., 2010).

A produção de micropartículas sem a utilização de solventes orgânicos torna a técnica de extrusão crescente e promissora, especialmente para a encapsulação de fármacos, células vivas imobilizadas e para inclusão de compostos de interesse em alimentos (Patil et al., 2010). O esquema do método de microencapsulação por extrusão encontra-se na figura 1:
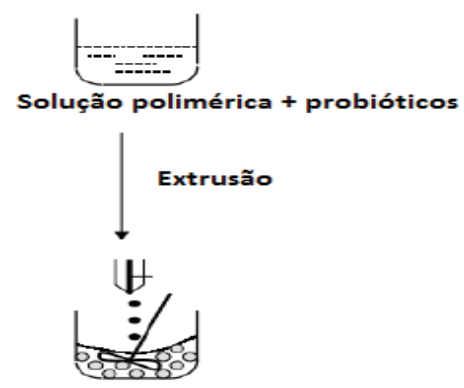

Solução de Cloreto de cálcio ou quitosana

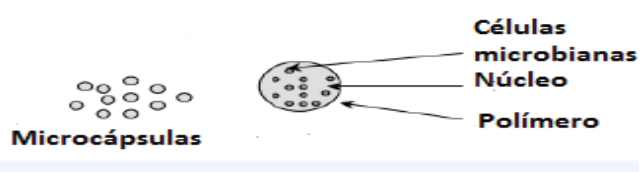

Figura 1: Esquema do método de extrusão (adaptado de: Krasaekoopt, Bhandari \& Deeth, 2000).

Em presença de cálcio e outros íons divalentes, o polissacarídeo pode, através de um processo de ligação cruzada, trocar o íon de sua estrutura por um íon divalente, geleificando o meio em que se encontra. Os cátions monovalentes e o íon $\mathrm{Mg} 2+$ não induzem a essa geleificação. Íons como $\mathrm{Pb} 2+, \mathrm{Cu} 2+, \mathrm{Cd} 2+, \mathrm{Co} 2+$, $\mathrm{Ni} 2+, \mathrm{Zn} 2+$ e Mn2+ possuem um uso limitado por apresentarem certa toxicidade (Krasaekoopt; Bhandari; Deeth, 2003).

Devido a essa propriedade de geleificação, o alginato de sódio, um dos polímeros mais utilizados na microencapsulação por extrusão, pode ser usado como matriz e assim envolver moléculas de significância biológica, tais como, enzimas, plantas, células animais e medicamentos (Valenga, 2007). O alginato de sódio gelifica após ligação dos íons bivalentes de cálcio aos blocos de ácido gulurônico das cadeias de alginato formando uma rede tridimensional, constituindo o modelo da "caixa de ovos", conforme figura 2 (Gombotz $\&$ Wee, 1998).

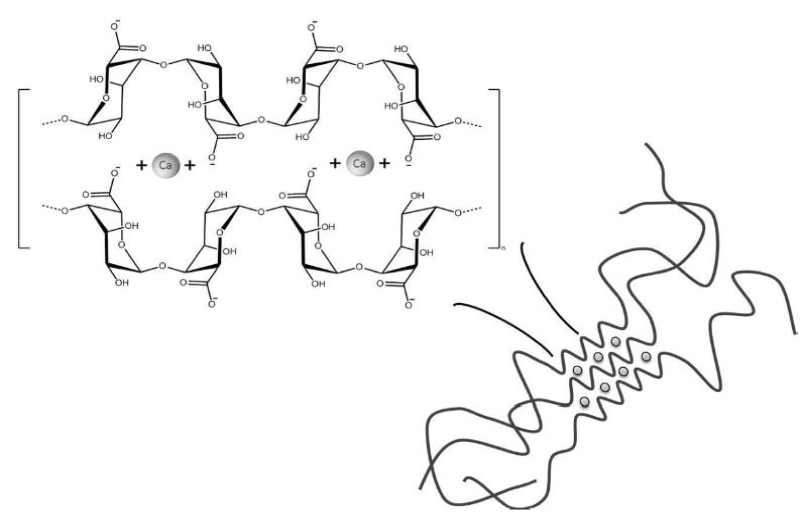

Figura 2: Modelo caixa de ovos (Fonte: CoronaHernandez et al. 2013)

Partículas produzidas por extrusão normalmente apresentam diâmetros que podem variar de $500 \mu \mathrm{m}$ a $3 \mathrm{~mm}$ (Burey et al., 2008; Krasaekoopt et al., 2003) sendo que o tamanho das partículas formadas é dependente do tamanho do diâmetro da agulha usada para gotejar a solução, da viscosidade e concentração da solução de alginato, e da distância entre a seringa e a solução de cloreto de cálcio (Krasaekoopt et al., 2003). Maiores diâmetros, viscosidades e concentrações mais altas produzem partículas maiores (Gacesa, 1988).

Por estes fatos, foram desenvolvidas variantes do método. Algumas destas consistem na utilização de um sistema de pressão para forçar a saída do alginato através de um aerógrafo para dispersar as gotas da extremidade com o qual se obtiveram partículas com menos de $70 \mu \mathrm{m}$, conforme figura 3 (Etchepare et al., 2016):

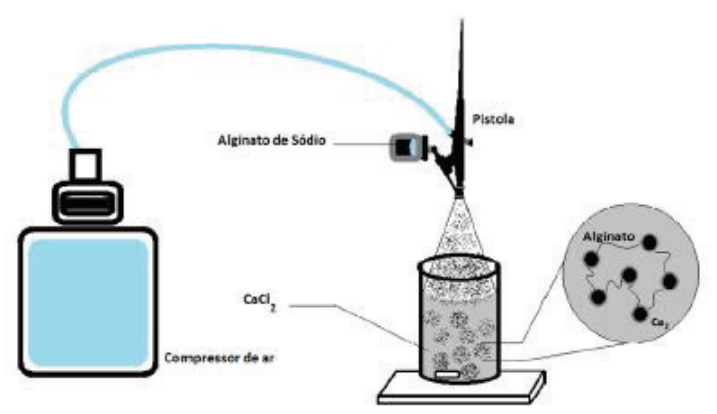

Figura 3: Esquema do processo de microencapsulação utilizando um aerógrafo acoplado a um compressor de ar (Etchepare et al. 2016). 
Para aplicações em alimentos, o diâmetro médio das micropartículas, é uma das características mais importantes, sendo que estas devem ser suficientemente pequenas, para evitar um impacto sensorial negativo, sendo o tamanho desejável de aproximadamente $100 \mu \mathrm{m}$ (Heidebach et al., 2009).

Pinpimai et al. (2015), estudaram a viabilidade de Saccharomyces cerevisiae encapsuladas em alginato por extrusão que serviram para suplementar a dieta de tilápias. Os autores avaliaram a viabilidade, morfologia, passagem pelo trato gastrointestinal, durante $\mathrm{o}$ armazenamento por 14 dias. A viabilidade das leveduras encapsuladas foi significativamente elevado em condições gástricas e biliares simuladas e manteve-se elevada após o armazenamento à temperatura ambiente durante os 14 dias avaliados.

Sousa et al. (2015) a fim de estudar o efeito do congelamento sobre a estabilidade das micropartículas de alginato de cálcio de Lactobacillus casei, Lactobacillus paracasei, Lactobacillus acidophilus e Bifidobacterium animalis, obtidas por extrusão, foram caracterizados no momento da produção e após 60 dias de armazenamento a $-20{ }^{\circ} \mathrm{C}$. Um aumento no tamanho das partículas, a perda de forma esférica e porosa foram observados depois do período de armazenagem. As micropartículas de $B$. animalis foram mais resistentes, tanto para o processo de microencapsulação, como para os testes de simulação gastrointestinais.

Uma desvantagem das cápsulas produzidas com polissacarídeo pela técnica de extrusão é a formação de poros na superfície, tornando-as sensíveis as condições ácidas do meio (Mortazavian et al., 2007). Para suprir esse problema, alguns pesquisadores têm recoberto as cápsulas usando uma combinação desse polissacarídeo com outros compostos poliméricos, a fim de garantir um maior efeito protetor aos microrganismos (Chávarri et al., 2010).

\section{Interações eletrostáticas}

A interação eletrostática ocorre quando biopolímeros que possuem cargas opostas interagem entre si. Normalmente, os sistemas de biopolímeros utilizados contêm uma molécula protéica como polieletrólito positivo e uma molécula de polissacarídeo como polieletrólito negativo Neste contexto, surgiu o método chamado de layer-by-layer que foi aplicado à área de microencapsulação, para a produção de multicamadas de polieletrólitos. A técnica fundamenta-se quando ocorre a adsorção consecutiva de camadas alternadas de polieletrólito carregado positivamente e negativamente sobre uma partícula modelo e tem como força matriz a interação eletrostática (Kreft et al., 2007; Jun-xia et al., 2011).

Policátions, como por exemplo, a quitosana, poli lisina, e proteínas do soro de leite, além de diminuírem a porosidade do gel, formam um complexo forte com alginatos que são estáveis na presença de agentes quelantes de $\mathrm{Ca}^{2+}$. A agregação destes compostos com alginato de cálcio leva ao desenvolvimento de cápsulas mais estáveis permitindo a formação de uma dupla parede na microcápsula (Calamaril, 2004; Gombotz et al., 1998).

O efeito de micropartículas de alginato revestidas com três tipos de quitosanas de diferentes pesos moleculares na sobrevivência de Lactobacillus bulgaricus KFRI 673 em suco gástrico simulado, sucos intestinais e estabilidade durante o armazenamento a 4 e $22^{\circ} \mathrm{C}$ foi investigado por Lee et al. (2004). Os autores concluíram que a microencapsulação com alginato e quitosana oferece um melhor revestimento e um meio mais eficaz de entrega bacteriana viável para o cólon, além de manter a sobrevivência durante o armazenamento refrigerado, principalmente com quitosana de alto peso molecular. A quitosana pode atuar como meio de revestimento das partículas, alterando o grau de difusão de substâncias encapsuladas ou como um aditivo para modificar a estrutura da partícula (Pasparakis \& Bouropoulos, 2006).

Gbassi et al. (2009) utilizaram proteína de soro de leite para recobrir micropartículas de alginato contendo culturas probióticas de Lactobacillus plantarum. Em testes em sistema gástrico simulado, as cápsulas com revestimento apresentaram uma proteção muito mais efetiva na viabilidade probiótica, do que as cápsulas sem o revestimento de proteína de soro de leite, demonstrando que este material é eficiente para o recobrimento de microcápsulas com intuito de maior viabilidade celular.

De Prisco et al. (2015), encapsularam Lactobacillus reuteri em alginato (MC) e em um 
complexo de quitosana-alginato (CMC) pela técnica de extrusão com o objetivo de melhorar a sua tolerância a ambientes adversos. Os autores obtiveram um rendimento de $97 \%$ de encapsulação e o estudo comprovou que as microcápsulas a base de alginato melhorou a sobrevivência de $L$. reuteri durante o armazenamento e a exposição a trato gastrointestinal e condições de estresse osmótico, concluíram ainda que, o co-encapsulamento do probiótico em quitosana-alginato, aumentou a sobrevivência dos probióticos.

Krasaekoopt \& Watcharapoka (2014) encapsularam Lactobacillus acidophilus e Lactobacillus casei em galactooligossacarídeo e inulina em cápsulas de alginato revestidas com quitosana pela técnica de extrusão. As cápsulas foram adicionadas em iogurte e sumo de fruta e mantiveram a viabilidade durante armazenamento refrigerado durante 4 semanas.

Ribeiro et al. (2014), encapsularam Lactobacillus acidophilus por combinação de gelificação iônica pela técnica de extrusão com recobrimento de pectina e proteínas do soro do leite. Os autores concluíram que a técnica tem potencial para entrega de probióticos ao trato gastrointestinal humano, uma vez que protegeu os microrganismos durante o armazenamento em iogurte conferindo menor acidificação e fornecendo um produto com uma boa impressão geral.

Omar et al. (2013), microencapsularam Lactocabillus amylovorus e Lactobacillus Fermentarum em cápsulas de Alginato com multicamadas, revestidas com $0,1 \%(\mathrm{w} / \mathrm{v})$ de poli-L-lisina e revestidas novamente com $0,1 \%$ $(\mathrm{w} / \mathrm{v})$ de alginato de sódio. Essas microcápsulas foram adicionadas em iogurte e utilizadas em um estudo randomizado que avaliou a perda de peso em pacientes obesos após o consumo do iogurte com microcápsulas probióticas, havendo mudanças na composição corporal após a ingestão.

Albadran et al. (2015) microencasularam Lactobacillus plantarum em alginato com recobrimento de quitosana, analisaram duas formas de secagem (liofilização e leito fluidizado) e avaliaram a viabilidade probiótica durante 45 dias em diferentes temperaturas (4, 30 e $\left.37^{\circ} \mathrm{C}\right)$. Os resultados indicaram que a secagem em leito fluidizado é um método de secagem eficiente e eficaz para a produção de cápsulas probióticas armazenadas.

Amine et al. (2014) avaliaram a viabilidade de Bifidobacterium longum microencapsulado por extrusão, utilizando diferentes concentrações de alginato nativo e s-palmitoilado como materiais de revestimento. Os autores concluíram que a recuperação de probióticos durante o encapsulamento é maior se o alginato é modificado por palmitoilação.

Tomas et al. (2015) utilizaram a técnica de extrusão para encapsular Lactobacillus reuteri, utilizando goma xantana e goma gelana como materiais de revestimento e a liofilização para a secagem das microcápsulas com e sem a adição de crioprotetores como lactose e leite desnatado. Após liofilização, as microcápsulas foram armazenadas à temperatura ambiente e à temperatura de refrigeração $\left(4^{\circ} \mathrm{C}\right)$ no escuro, por 150 dias. A sobrevivência de $L$. reuteri encapsulado durante a liofilização e armazenagem foi significativamente maior na presença de crioprotetores.

Divya et al. (2015), avaliaram a microencapsulação de Lactococcus lactis pela técnica de extrusão em alginato e manitol com leite desnatado, glicerol, $\mathrm{CaCO} 3$ a adicionaram em alimento fortificado com folato. Os autores concluíram que a utilização de leite desnatado, glicerol, $\mathrm{CaCO}_{3}$ e alginato proporcionou melhor proteção para cepas L. lactis que foram capazes de fermentar o ácido fólico em leite desnatado. Além de sobreviverem melhor as condições gastrointestinais simuladas em comparação com as células livres.

A sobrevivência de Lactobacillus rhamnosus encapsulado em diferentes concentrações de bagaço de cana de açúcar e alginato de sódio por extrusão foi avaliada através de exposição a tratamento térmico de $90{ }^{\circ} \mathrm{C}$ por $30 \mathrm{~s}$. Os resultados demonstraram um grande potencial na formação de microcápsulas probióticas resistentes ao calor (shaharrudin et al. 2015).

Alves et al. (2015), microencapsularam Lactobacillus plantarum em cápsulas de alginato com adição de Hi-maize e aplicaram em pasta de azeitona. Este estudo concluiu que as microcápsulas não comprometeram os atributos sensoriais e propriedades texturais da pasta de azeitona tornando-se um produto eficiente para veicular probióticos. Em relação às microcápsulas, os autores confirmam que a 
adição do Hi-maize não influenciou a sobrevivência dos microganismos durante o armazenamento refrigerado.

O microrganismo Enterococcus durans foi microencapsulado por extrusão em nove tratamentos de alginato-psyllium misturados com diferentes concentrações de prebióticos (inulina ou fenogrego). Os objetivos deste estudo foram de aumentar a sua taxa de sobrevivência em condições gastrointestinais durante o tempo de armazenamento. A maior eficiência de encapsulação, a viabilidade das células em condições gastrointestinais durante o tempo de armazenamento e também as maiores taxas de liberação de células probióticas em condição colônicas ao longo do período de $12 \mathrm{~h}$ foi observada para o tratamento de alginatopsyllium com 2\% de feno-grego na formulação (Haghshenas et al., 2015).

Fareez et al. (2015) microencapsularam por extrusão Lactobacillus prantarum em alginato, alginato + quitosana, alginato+goma xantana+ alginato+goma xantana+quitosana. As microcápsulas de Alginato+ goma xantana+ quitosana melhoraram a sobrevivência de Lactobacillus plantarum nos testes gastrointestinais, na resistência térmica a $90^{\circ} \mathrm{C}$, e no tempo de armazenamento a $4{ }^{\circ} \mathrm{C}$ por 4 semanas. Estes bons resultados são atribuídos as propriedades físico-químicas das microcápsulas onde a inclusão destes três componentes polimeréricos resultou em boa miscibilidade com nenhuma mudança no tamanho do grânulo quando comparado com os outros tratamentos.

Shi et al. (2013) encapsularam Lactobacillus bulcaricus em microcápsulas de leite revestidas de goma de alfarroba e carragena com o objetivo de aumentar a sobrevivência durante a exposição a condições ambientais adversas do trato gastrointestinal. Os resultados indicaram que as microcápulas de leite com estrutura de dupla camada podem melhorar a estabilidade do $L$. bulgaricus em condições gastrointestinais simuladas, assim como reduzir a porosidade da mesma.

Pirarat et al. (2015) pruduziram microcápuslas com Lactobacillus rhamnosus de alginato e alginato com leite desnatado pela técnica de extrusão e compararam com células livres após suplementação de dietas para tilápias Os resultados in vitro mostraram que a viabilidade das células, tanto da matriz de alginato como na matriz de alginato com leite desnatado foram mantidas depois de 14 dias de armazenamento a temperatura ambiente, enquanto Lactobacillus rhamnosus livre não foram viáveis após 7 dias. A microencapsulação de probióticos com alginato em combinação com $10 \%$ de leite desnatado é um método adequado para melhorar a viabilidade dos probióticos durante o armazenamento e em caso de exposição a condições gastrointestinais de tilápias, o microrganismo encapsulado pode ser aplicado para melhorar o desempenho do crescimento e reduzir a mortalidade em tilápias.

Lactobacillus casei foi encapsulado em microesferas de alginato de sódio e pectina em diferentes concentrações pela técnica de extrusão e adicionadas em iogurte. Os autores concluíram que, a utilização de misturas binárias pectinaalginato permite a obtenção de microcápsulas que melhoram a proteção de $L b$. Casei contra fatores ambientais adversos do que aquelas microcápsulas desenvolvidas unicamente com alginato ou pectina. As propriedades de textura, e a eficácia de encapsulação de pectina-alginato são bastante afetadas pela concentração total do biopolímero assim como a relação entre ambos (Sandoval Castila et al., 2010).

A encapsulação por extrusão em goma xantana e quitosana aumentou as taxas de sobrevivência das células contra a liofilização e em fluidos gástrico simulados. Estes resultados sugerem que cápsulas de goma xantana e quitosana tem um bom potencial para a entrega de probióticos a nível intestinal em número elevado (Argin et al., 2014).

Vários polímeros microbianas, como goma xantana, goma gelana, goma de pululano e jamilan, foram testados como materiaias de revestimento de encapsulação para Lactobacillus plantarum and Lactobacillus rhamnosus A proteção oferecida pelos polímeros microbianas específicas utilizado neste trabalho para as cepas de lactobacilos em avaliação, principalmente a mistura polimérica de xantana e goma gelana ( $1 \%$ e $0,75 \%$ respectivamente), mostrou ser mais resistentes em condições gastrointestinais simulada. Assim, polímeros microbianos parece ser uma interessante fonte de material de revestimento para a encapsulação de probióticos (Jimenez-Pranteda et al. 2012).

$\mathrm{Ma}$ et al. (2012) relatam que a microencapsulação de bacteriófago $\mathrm{K}$ em 
alginato por extrusão, melhorou sua sobrevivência em relação aos bacteriófago livre em exposição a ambiente gastrointestinal simulado. No entanto, a co-encapsulação de partículas de alginato com carbonato de cálcio proporcionou uma melhor proteção contra ambientes ácidos. Este efeito protetor melhorado pode ser atribuído à força aumentada de redes de gel de alginato e um microclima mais neutro dentro das microesferas.

O efeito de diferentes métodos de microencapsulação (extrusão e emulsão), diferentes concentrações de inulina $(0,1 \%$ e $2 \%$ ) e resistência gastrointestinal sobre a viabilidade do Lactobacillus plantarum durante o armazenamento $(0,15$ e 30 dias $)$ a $4^{\circ} \mathrm{C}$ foi investigado. Os autores concluiram que o tamanho das microcápsulas foi afetado pelo método de encapsulamento e pela a concentração de inulina. Os resultados durante o armazenamento demonstraram a melhor sobrevivência de L. plantarum com a utilização de $2 \%$ de inulina. No final dos testes de rsistência gastrointestinal em ambos os métodos de microencapsulação, as microcápsulas de $L$. plantarum tiveram uma alta sobrevivência, acima do mínimo terapêutico recomendado $\left(>10^{6}\right.$ UFC $\left.\mathrm{g}^{-1}\right)$, embora a partir de 15 dias de armazenamento, as cápsulas microencapsuladas por emulsão interna não resistiram à digestão gástrica, perderam a sua integridade e liberaram o microrganismo para o meio externo. Assim, a microencapsulação por extrusão foi o melhor método para proporcionar uma maior proteção e sobrevivência para o L plantarum microencapsulado durante os períodos de armazenamento (Vallero Cases et al., 2015).

\section{Conclusões}

A microencapsulação de probióticos pelo método de extrusão associado a interações eletrostáticas vem sendo bastante explorada atualmente e tem mostrado resultados positivos e promissores no aumento da viabilidade das bactérias probióticas, sendo uma técnica simples e de baixo custo, que pode ser explorada para melhorar a viabilidade e proteção dos microrganismos durante o processamento e armazenamento de alimentos, bem como passagem pelo trato gastrointestinal, em comparação com bactérias livres. Nesta revisão, percebe-se a grande variedade de materiais de revestimento utilizados nos estudos de extrusão com grande potencial de proteção, embora o alginato de sódio seja utilizado com mais freqüência, nota-se que a viabilidade probiótica depende de uma série de fatores como, por exemplo, o tipo e a concentração do material de revestimento, da interação dos materiais quando usados em conjunto, e também, do tipo de microrganismo utilizado.

\section{Agradecimentos}

Pelo apoio financeiro ao Conselho Nacional de Desenvolvimento Científico e Tecnológico (CNPq).

\section{Referências}

Abdullah, N. (2015). Effect of addition of inulin and fenugreek on the survival of microencapsulated Enterococcus durans $39 \mathrm{C}$ in alginate-psyllium polymeric blends in simulated digestive system and yogurt. Asian Journal of pharmaceuticalsciences, 10, 350-361.

Albadran, H. A., Chatzifragkou, A., Khutoryanskiy, A. V., Charalampopoulos, D. (2015). Stability of probiotic Lactobacillus plantarum in dry microcapsules under accelerated storage conditions. Food Research International, 74, 208-216.

Albertini, B., Vitali, B., Passerinia, N., Crucianib, F.; Di Sabatinoa, M., Rodrigueza, L., Brigidi, P. (2010). Development of microparticulate systems for intestinal delivery of Lactobacillus acidophilus and Bifidobacterium lactis. European Journal of Pharmaceutical Sciences, 40, 359-366.

Altamirano-Fortoul, R., Moreno-Terrazas, R., Quezada-Gallo, A., Rosell, C. M. (2012). Viability of some probiotic coatings in bread and its effect on the crust mechanical properties. Food Hydrocolloide, 29, 166-74

Alves, M., Peres, C. M., Hernandez-Mendonza, A., M. Bronze, M. R., Peres , C., Malcata, F., X. (2015). Olive paste as vehicle for delivery of potential probiotic Lactobacillus plantarum 33. Food Research International, 75, 61-70.

Amine, K. M., Champagne, C. P., Salmieri, S., Britten, M., St-Gelais, D., Fustier, P., Lacroix, M. (2014). Effect of palmitoylated alginate microencapsulation on viability of Bifidobacterium longum during freeze-drying. LWT - Food Science and Technology, 56, 111-117. 
Argin, S., Kofinas, P., Lo, Y. M. (2014). The cell release kinetics and the swelling behavior of physically crosslinked xanthanechitosan hydrogels in simulated gastrointestinal conditions. Food Hydrocolloids, 40, 138-144.

Barbosa, Y., M'arquez, E., Parra, K., Piñeiro, M.P., Medina LM. (2012). Development of a potential functional food prepared with pigeon pea (Cajanus cajan), oats and Lactobacillus reuteri ATCC 55730. International Journal Food Science Nutrition 63(7), 813-820.

Brasil. Lista de alegações de propriedade funcional aprovadas. Brasília. (2012). Disponível em:<http://www.anvisa.gov.br/ alimentos/comissoes/tecno_lista_alega.htm $>$.

Burey, P., Bhandari, B. R, Howes, T., Gidley, M. J. (2008). Hydrocolloid Gel Particles: Formation, Characterization, and Application. Critical Reviews in Food Science and Nutrition, 48, 361-377.

Champagne, C. P., Fustier, P. (2007). Microencapsulation for the improved delivery of bioactive compounds into foods. Current Opinion in Biotechnology, 18(2), 184-190.

Chávarri, M., Marañón, I., Ares, r., Ibáñez, F. C., Marzo, F., Villarán, M. C. (2010). Microencapsulation of a probiotic and prebiotic in alginate-chitosan capsules improves survival in simulated gastro-intestinal conditions. International Journal of Food Microbiology, 142, 185-189.

Cook, M. T., Tzortzis, G., Charalampopoulos, D. (2012). Microencapsulation of probiotics for gastrointestinal delivery. Journal of Controlled Release, 162, 56-67.

Corona-Hernandez, R. I., Parrilla, E. A., LizardiMendoza, J., Islas-Rubio, A. R., Rosa, L. A., Wall-Medrano, A. (2013). Structural Stability and Viability of Microencapsulated Probiotic Bacteria: A Review. Comprehensive Reviews in Food Science and Food Safety, 12, 614-628.

De Prisco, A., Maresca, D., Ongeng, D., Mauriello, G. (2015). Microencapsulation by vibrating technology of the probiotic strain Lactobacillus reuteri DSM 17938 to enhance its survival in foods and in gastrointestinal environment. LWT - Food Science and Technology, 61, 452-462.

Divya, J. B., Nampoothiri, K. M. (2015). Encapsulated Lactococcus lactis with enhanced gastrointestinal survival for the development of folate enriched functional foods. Bioresource Technology, 188, 226-230.

Etchepare, M. A., Raddatz, C. G., Flores, E. M. M., Zepka, L. Q., Jacob-Lopes, E., Barin, J. S., Grosso, C. R. F., Menezes, C. R. (2016). Effect of resistant starch and chitosan on survival of Lactobacillus acidophilus microencapsulated with sodium alginate. LWT - Food Science and Technology, 65, 511-517.

Fareez, I. M., Lim, S. M., Mishra, R. K., Ramasamy, K. (2015). Chitosan coated alginate-xanthan gum bead enhanced $\mathrm{pH}$ andthermotolerance of Lactobacillus plantarum LAB12. International Journal of Biological Macromolecules, 72, 1419-1428.

Fahimdanesh, M., Mohammadi, N., Ahari, H., Khosravi, M. A., Zanjani F. Z. H., Behrouznasab, K. 2012. Effect of microencapsulation plus resistant starch on survival of Lactobacillus casei and Bifidobacterium bifidum in mayonnaise sauce. African Journal Microbiology Research, 6(40), 6853-6858.

Food and Agriculture Organization of the United Nations; World Health Organization. (2001) Health and nutritional properties of probiotics in food including powder milk with live lactic acid bacteria.

Gacesa, P. (1988). Alginates. Carbohidrate Polymers, 8, 161-182.

Gawkowski, D., Chikindas, M. L. (2013). Nondairy probiotic beverages: the next step into human health. Beneficial Microbes, 4(2),127-42.

Gbassi, G. K. (2009) Microencapsulation of Lactobacillus plantarum spp in an alginate matrix coated with whey proteins. International Journal of Food Microbiology, 129, 103-105.

Gombotz, W. R., We, S. F. (1998). Protein release from alginate.Advanced Drug Delivery Reviews, 31, 67-285.

Gomes, A. A., Braga, S. P., Cruz, A. G., Cadena, R., Sm Lollo, P. C. B., Carvalho, C., Amaya-Farfan, J., Faria, J. A. F., Bollini, H. M. A. (2011). Effect of the inoculation level of Lactobacillus acidophilus in probiotic cheese on the physicochemical features and sensory performance compared with commercial cheeses. Journal Dairy Science, 94, 4777-4786.

Haghshenas, B., Nami, Y., Haghshenas, M., Barzegari, A., Sharifi, S., Radiah, D., Rosli, R., Abdullah, N. (2015). Effect of addition of inulin and fenugreek on the survival of microencapsulated Enterococcus durans 39C in alginate-psyllium polymeric blends in simulated digestive system and yogurt. Asian Journal of pharmaceutical sciences, 10, 350-361. 
Heidebach, T. (2009). Microencapsulation of probiotics cells by means of rennet-gelation of milk proteins. Food hidrocolloids, 23, 1670-1677.

Ibarra, A., Acha, R., Calleja, M. T., Chiralt-Boix, A., Wittig, E. (2012). Optimization and shelf life of a low-lactose yogurt with Lactobacillus rhamnosus HN001. Journal Dairy Science, 95, 3536-3548.

Jackson, L. S.; Lee, K. (1991). Microencapsulation and Food Industry. LWT - Food Science and Technology, (24) 4, 289-297.

Jiménez-Pranteda, M. J., Poncelet, D., NáderMacías, M. E., Arcos, A., Aguilera, M., Monteoliva-Sánchez,M., Ramos-Cormenzana, R. (2012). Stability of lactobacilli encapsulated in various microbial polymers, Journal of Bioscience and Bioengineering, 113(2), 179-184.

Jun-Xia, X., Hai-Yan, Y., Jian, Y. (2011). Microencapsulation of sweet orange oil by complex coacervation with soybean protein isolate/ gum Arabic. Food Chemistry, 125, 1267-1272.

Kim, S. C., Olson, N. F. (1989). Production of methanethiol in milk fat-coated microcapsules containing Brevibacterium linens and methionine. Journal of Dairy Research, 56(5) 799-811.

Kim, S. J., Chob, S. Y., Kim, S. H., Songd, O. J., Shind, I. S., Cha, D. S., Park, H.J. (2008). Effect of microencapsulation on viability and other characteristics in Lactobacillus acidophilus ATCC 43121. Food Science and Technology, 41, 493-500.

Krasaekoopt, w., Watcharapoka, S. (2014). Effect of addition of inulin and galactooligosaccharide on the survival of microencapsulated probiotics in alginate beads coated with chitosan in simulated digestive system, yogurt and fruit juice. LWT - Food Science and Technology, 57, 761-766.

Krasaekoopt, W., Bhandari, B., Deeth, H. (2003). The influence of coating materials on some properties of alginate beads and survivability of microencapsulated probiotic bacteria. International Dairy Journal, 14, 737-743.

Krasaekoopt, W., Bhandari, B., Deeth, H. (2003). Review: Evaluation of encapsulation techniques of probiotics for yoghurt. International Dairy Journal, 13, 3-13.

Kreft, O., Prevot, M., Mohwald, H., Sukhorukov, G. B. (2007). Shell-in-Shell. Microcapsules: A Novel Tool for Integrated, Spatially Confined Enzymatic
Reactions. Angew. Chem. Int., 46, 5605 -5608.

Lambert, J. M., Weinbreck, F., Kleerebezem, M. (2008). In vitro analysis of protection of the enzyme bile salt hydrolase against enteric conditions by whey protein - gum Arabic microencapsulation. Journal of Agricultural and Food Chemistry, 56, 8360- 8364.

Lee, S., Cha, D. S., Park, H. J. (2004). Survival of Freeze-Dried Lactobacillus bulgaricus KFRI 673 in Chitosan-Coated Calcium Alginate Microparticles. Journal Agricultural and Food Chemistry, 52(24), 7300-7305.

Ma, T., Pacan, J. C., Wang, Q., Sabour, P. M., Huang, X., Xu, Y. (2012). Enhanced alginate microspheres as means of oral delivery of bacteriophage for reducing Staphylococcus aureus intestinal carriage. Food Hydrocolloids, 26, 434-440.

Mattila-sandholm, T., Myllärinen, P., Crittenden, R.; Mogensen, G., Fondén, R., Saarela, M. (2002). Technological challenges for future probiotic foods. International Dairy Journal, 12, 173-182.

Menezes, C. R., Durrant, L. R. (2008). Xilooligossacarídeos: produção, aplicações e efeitos na saúde humana. Ciência Rural, 38, 587-592.

Mortazavian, A. M., Sohrabvandi, S. (2007). Probiotics and Food Probiotic Products. Eta Publication, 131-169.

Nazzaro, F.; Orlando, P.; Fratianni, F.; Coppola, R. (2011). Microencapsulation in food science and biotechnology. Current Opinion in Biotechnology, 23, 1-5.

Oliveira, A. C., Moretti, T. S., Boschini, C., Baliero, J. C. C., Freitas, O., Favaro-Trindade, C. S. (2007). Stability of microencapsulated B. lactis (BI 01) and L. acidophilus (LAC 4) by complex coacervation followed by spray drying. Journal of Microencapsulation, 24(7), 685-693.

Omar, J. M., Chan, Y., Jones, M. L., Prakash, S., Jones, P. J. H. (2013). Lactobacillus fermentum and Lactobacillus amylovorus as probiotics alter body adiposity and gut microflora in healthy persons. Journal of functional foods, 5, 116 -123.

Pasin, B. L., Azón, C. G., Garriga, A. M. (2012). Microencapsulación con alginato en alimentos. Técnicas y aplicaciones. Revista Venezolana de Ciencia y Tecnología de Alimentos, 3(1), 130-151.

Pasparakis, G. N., Bouropoulos, N. Swelling studies and in vitro release of verapamil from calcium alginate 
and calcium alginate-chitosan beads. International Journal Pharmaceutics, 323, 34-42, 2006.

Pasqualim, P., Culpi, T.A.; Kaminski, G. A. T.; Fin, M. T.; Sasso, D. G. B., Costa, C. K., Miguel, M. D., Fujiwara, G. M., Rodrigues, B. H., Zanin, S. M. W. (2010).Microcapsules of calcium alginate and vegetable oil by ionic gelation: a study of encapsulation capacity and skin application. Visão Acadêmica, Curitiba, 11(1).

Patil, J. S., Kamalapur, M. V., Marapur, S. C., Kadam, D. V. (2010). Ionotropic gelation and polyelectrolyte complexation: The novel techniques to design hydrogel particulate sustained, modulated drug delivery system: A review. Digest Journal of Nanomaterials and Biostructures, 5(1), 241-248.

Pedroso, D. D. L., Thomazini, M., Heinemann, R. J. B., Favaro-trindade, C. S. (2012). Protection of Bifidobacterium lactis and Lactobacillus acidophilus by microencapsulation using spraychilling. International Dairy Journal, 26(2), 127-132.

Pinpimai, K., Rodkhum, Chansue, N., Katagiri, T., Maita, M., Pirarat, N. (2015). The study on the candidate probiotic properties of encapsulated yeast, Saccharomyces cerevisiae JCM 7255, in Nile Tilapia (Oreochromis niloticus). Research in Veterinary Science 102, 103-111.

Pirarat, N., Pinpimai, K., Rodkhum, C., Chansue, N., Ooi, E. L., Katagiri, T., Maita, M. (2015). Viability and morphological evaluation ofalginate-encapsulated Lactobacillus rhamnosus GG undersimulated tilapia gastrointestinal conditions and its effect ongrowth performance, intestinal morphology and protectionagainst Streptococcus agalactiae. Animal Feed Science and Technology, 207, 93-103.

Racoviță, S., Vasiliu, S., Popa, M., Luca, C. (2009). Polysaccharides based on micro- and nanoparticles obtained by ionic gelation and their applications as drug delivery systems. Revue Roumaine de Chimie, 54(9), 709-718.

Ribeiro, M. C. E., Chaves, K. S., Gebara, C., Infante, F. N. S., Grosso, C. R. F., Gigante, M. L. (2014). Effect of microencapsulation of Lactobacillus acidophilus LA- 5 on physicochemical, sensory and microbiological characteristics of stirred probiotic yoghurt. Food Research Internationa, 166, 424-431.

Sandoval-Castilla, O., Lobato-Calleros, C., GarcíaGalindo, H. S., Alvarez-Ramírez, J., Vernon-Carter, E. J. (2010). Textural properties of alginate-pectin beads and survivability of entrapped Lb. casei in simulated gastrointestinal conditions and in yoghurt. Food Research International, 43,111-117.

Shah, N. P. (2007). Functional cultures and health benefits. International Dairy Journal, 17,1262-1277.

Shaharuddin, S., Muhamad, I., I. (2015). Microencapsulation of alginate-immobilized bagasse with Lactobacillus rhamnosus NRRL 442: Enhancement of survivability and thermotolerance. Carbohydrate Polymers, 119, 173-181.

Sheu, T. Y., Marshall, R. T. (1993). Microentrapment of lactobacilli in calcium aginate gels. Journal of Food Science, 54(3), 557-561.

Shi, L. E., Li, Z. H., , Zhang, Z. L., Zhang, T. T., Yu, W. M., Zhou, M. L., Tang, Z. X. (2013). Encapsulation of Lactobacillus bulgaricus in carrageenan-locust bean gum coated milk microspheres with double layer structure. LWT - Food Science and Technology, 54, 147-151.

Smrdel, P. (2008). Shape optimization and characterization of polysaccharide beads prepared by ionotropic gelation. Journal of Microencapsulation, 25(2)90-105.

Sousa, S., Gomes, A. M., Pintado, M. H., Silva, J. P., Costa, P., Amaral, M. H., Duarte, A. C., Rodrigues, D., Rocha-Santos, T. A. P., Freitas, A. C. (2015). Characterization of freezing effect upon stability of probiotic loaded, calcium-alginate microparticles. Food and Bioproducts Processing, 93, 90-97.

Sultana, K., Godward, G., Reynolds, N., Arumugaswamy, R., Peiris, P., Kailasapathy, K. (2000). Encapsulation of probiotic bacteria with alginate-strach and evaluation of survival in simulated gastrointestinal conditions and in yogurt. International Journal of Food Microbiology, 62, 47-55.

Tomás, M. S. J., Gregorio, P. R., Terraf, M. C. L., Nader-Macıas, M. E. F. (2015). Encapsulation and subsequent freeze-drying of Lactobacillus reuteri CRL 1324 for its potential inclusion in vaginal probiotic formulations. European journal of pharmaceutical sciences, 79,87-95.

Trujillo-de Santiago, G., Saenz-Collins, C. P., Rojas-de Gante, C. (2012). Elaboration of a probiotic oblea from whey fermented using Lactobcillus acidophilus or Bifidobacterium. Journal Dairy Science, 95, 6897-904.

Valenga, F. (2007). Estudos de interação entre galactomanana e alginato e possíveis aplicações. 90 f. Dissertação (Mestrado 
em Bioquímica) - Centro de Ciências Biológicas, Universidade Federal do Paraná.

Valero-Cases, E., Frutos, M. J. (2015). Effect of different types of encapsulation on the survival of Lactobacillus plantarum during storage with inulin and in vitro digestion. LWT - Food Science and Technology, 64, 824-828.

Zanjani, M. A. K., Tarz, B. G., Sharifan, A., Mohammadi, N., Bakhoda, H., Madanipour, M. M. (2012). Microencapsulation of Lactobacillus casei with calcium alginate-resistant starch and evaluation of survival and sensory properties in cream-filled cake. African Journal Microbiology Research, 6(26), 5511-5517. 